\title{
Erratum to: Immature Dendritic Cell-Derived Exosomes: a Promise Subcellular Vaccine for Autoimmunity
}

\author{
Weifan Yin, ${ }^{1}$ Song Ouyang, ${ }^{2}$ Yi Li, ${ }^{1}$ Bo Xiao, ${ }^{1}$ and Huan Yang $^{1,3}$
}

Erratum to: Inflammation

DOI 10.1007/s10753-012-9539-1

The original version of this article unfortunately contained a mistake. The Acknowledgment section below was left out by the author.

\section{ACKNOWLEDGMENTS}

This work was supported by the National Nature Science Foundation of China (No. 81070962).

The online version of the original article can be found at http:// dx.doi.org/:10.1007/s10753-012-9539-1.

${ }^{1}$ Department of Neurology, Xiangya Hospital, Central South University, Changsha, Hunan, People's Republic of China 410008

${ }^{2}$ Department of Neurology, The First Hospital in Changsha City, Changsha, Hunan, People's Republic of China

${ }^{3}$ To whom correspondence should be addressed at Department of Neurology, Xiangya Hospital, Central South University, Changsha, Hunan, People’s Republic of China 410008. E-mail: yangh69@126.com 